\title{
La libération des otages allemands au Liban : analyse politique et judiciaire (partie 2)
}

Sylvie Lemasson

\section{OpenEdition}

1 Journals

\section{Édition électronique}

URL : http://journals.openedition.org/conflits/758

DOI : $10.4000 /$ conflits. 758

ISSN : $1777-5345$

Éditeur :

CCLS - Centre d'études sur les conflits lilberté et sécurité, L'Harmattan

\section{Édition imprimée}

Date de publication : 21 mai 1992

ISSN : 1157-996X

\section{Référence électronique}

Sylvie Lemasson, "La libération des otages allemands au Liban : analyse politique et judiciaire (partie 2) », Cultures \& Conflits [En ligne], 05 | printemps 1992, mis en ligne le 07 janvier 2003, consulté le 30 mars 2021. URL : http://journals.openedition.org/conflits/758 ; DOI : https://doi.org/10.4000/conflits 758

Ce document a été généré automatiquement le 30 mars 2021.

Creative Commons License 


\title{
La libération des otages allemands au Liban : analyse politique et judiciaire (partie 2)
}

\author{
Sylvie Lemasson
}

Les rouages diplomatiques et les contacts privés

Dans la capitale fédérale, le ministère des Affaires étrangères abrite un état-major de crise composé d'experts (diplomates hommes politiques), de conseillers (chercheurs) et de membres des services de sécurité. Hans-Dietrich Genscher mène une diplomatie qu'il qualifie de "silencieuse", mais active, à l'égard des ravisseurs déclarés (Hezbollah/"clan" Hamade) et des états impliqués (Syrie, Iran). Il confiera notamment à des médiateurs expérimentés, comme Hans-Jürgen Wischnewski, des missions sur le terrain. L'ancien ministre social-démocrate de Helmut Schmidt, mais avant tout l'homme de contacts incontournable pour les situations délicates entre la RFA et le Moyen-Orient ${ }^{1}$, sera dépêché à Téhéran en mai 1987, pour s'entretenir du problème des Otages $^{2}$. L'Auswärtiges Amt disposera également d'émissaires gouvernementaux en la personne du secrétaire d'état aux Affaires étrangères, Jürgen Möllemann, homme de terrain et arabophile. Par ailleurs, Hans-Dietrich Genscher s'appuiera sur une personnalité clé, Sadegh Tabatabai, proche de la famille Khomeyni et diplomate iranien, afin de resserrer les contacts avec la Syrie, les ravisseurs libanais et l'Iran. Tabatabai, qui se rend à Beyrouth et à Damas, en septembre 1987, a déjà eu l'occasion de tenir un rôle de médiateur avec la RFA, dans l'affaire des otages américains retenus dans leur ambassade à Téhéran en 1980.

Bien que la RFA dénie toute remise de rançons et affirme n'avoir "rempli aucune condition", il est néanmoins légitime de s'interroger sur le "prix"3 ou le contenu des négociations, dans la mesure où des intermédiaires libanais clairement identifiés, comme Hachid Maroun, ne seraient pas intervenus sans contrepartie. De plus, la constance des bonnes relations avec l'Iran ainsi que la normalisation avec la Syrie contribuent directement à la libération des otages. Nous avons vu en effet que la politique européenne de Hans-Dietrich Genscher visait à redonner du crédit à l'Iran et à 
la Syrie auprès de la CE. Tant que celle-ci n'a pas assuré son soutien financier, la RFA l'a fait seule par une aide à Damas de 146,1 millions de DM, dont 75,5 millions auraient dépendu $\mathrm{du}$ sort des otages ${ }^{4}$. Outre l'argent, en adressant officiellement ses remerciements "au président Hafez el Assad, au président de l'Assemblée nationale iranienne Rafsandjani et au ministre des Affaires étrangères de la République d'Iran, Ali Akbar Velayati", les autorités fédérales rendent explicite l'influence de Damas et de Téhéran sur les ravisseurs mais témoignent également, sinon d'accords, du moins d'ententes avec les deux capitales. Parallèlement aux prises de position en faveur de la Syrie et de l'Iran, le chef de la diplomatie de la République fédérale a en effet invité les industriels allemands à participer à la restructuration économique iranienne : marché potentiel immense pour les investissements après huit années de guerre contre l'Irak. Ainsi, par ses encouragements, Hans-Dietrich Genscher s'assure la confiance et le soutien de Téhéran qui annoncera notamment à Bonn la date de la libération de Rudolf Cordes. D'ailleurs, en soulignant le fait que le "maintien de relations diplomatiques avec l'Iran en des temps difficiles a fini par payer", le ministre d'outre-Rhin met en évidence une politique d'ensemble qui a permis la libération des ressortissants allemands même si sa conception générale dépasse le cas ou la problématique spécifique du règlement des otages.

Si Hans-Dietrich Genscher et le chancelier Kohl démentent que toute rançon ait été versée par des réseaux officiels, il est en revanche permis de penser que cette rançon a été effectuée sur une base non officielle. Ce dernier procédé présente différents avantages et s'appuie sur une réalité concrète. Il permet au gouvernement de ne pas être impliqué directement dans des tractations de nature financière avec les ravisseurs qui affaibliraient ses positions ultérieures face aux terroristes et qui susciteraient la relance de chantage à l'encontre des autorités étatiques en général. En revanche pour payer le million de dollars dont font état les ravisseurs pour la libération de Alfred Schmidt, puis exigée pour celle de Rudolf Cordes 5 , les firmes responsables des deux ressortissants ont été très probablement sollicitées. Siemens et Hoechst symbolisent en effet la force commerciale de la RFA implantées dans la région mais aussi la capacité financière à répondre à une des revendications classique de la panoplie du chantage.

Par ailleurs, l'implication de Franz-Joseph Strauss, ministre-président de Bavière, dans la libération de Alfred Schmidt induit deux réflexions. Elle corrobore le volet économico-commercial dans la conduite de négociations et la multiplicité de contacts entrepris avec le Hezbollah et les états "Parrains", au risque de concurrencer la diplomatie du gouvernement fédéral au profit des propres intérêts politiques du chef de la CSU. Grâce à ses rapports très étroits avec le ministre de la Défense syrien, le général Tlass, et ses contacts personnels avec le président Hafez el Assad, Franz Joseph Strauss revendique, en 1987, une partie de la responsabilité dans la libération du premier otage allemand. Cette revendication repose sur des liens particuliers avec le régime syrien, noués à travers le règlement de contrats commerciaux, en particulier dans le domaine des produits oléagineux et de l'armement. Au cours de l'année 1987, le dirigeant politique de Bavière dépêche, à Damas, son fils, Max, et un de ses proches, homme d'affaires d'envergure internationale, Dieter Holzer, afin de conclure des arrangements économiques susceptibles de constituer une base solide d'intérêts mutuels. Ces pratiques se concrétisent parallèlement aux efforts entrepris par le Gouvernement. Mais lorsque Franz-Joseph Strauss assure à Hafez el Assad qu'un des frères Hamade serait libéré à court terme, il met, à son tour en exergue, un des points cruciaux du panier des négociations : le volet juridique. 
Les enjeux des procès Hamade

$\mathrm{Au}$ cours du procès de Abbas Hamade, le gouvernement de Bonn reconnaît publiquement avoir cédé aux pressions des preneurs d'otages. Lors de son audition, le ministre de la Chancel1erie Wolfgang Schäuble, qui dirige une cellule de crise sur l'affaire déclare que les responsables politiques allemands "ont décidé de ne pas extrader (Mohamed Hamade) après avoir soupesé tous les risques à la lumière des menaces planant sur les otages Elles ont influencé notre décision"6. Les autorités gouvernementales avaient déjà choisi, en janvier 1988 en dépit de l'arrestation d'une jeune Allemande proche du "clan" Hindawi, de privilégier l'établissement d'une entente cordiale entre la Syrie et la RFA dans un climat pourtant lourd de soupçons concernant le soutien logistique des services secrets de Damas à l'organisation d'attentats ${ }^{7}$. Mais qu'en est-il de l'indépendance du pouvoir judiciaire dans le jugement d'actes terroristes ou quel est le degré d'interdépendance existant entre celui-ci et le pouvoir politique? Si le ministère de la Justice allemande décide de ne pas extrader Mohamed Ali Hamade, et facilite en cela la position de Bonn vis-à-vis des ravisseurs, il fonde son action sur le "principe universel de droit" (Weltrechtsprinzip). Ce dernier permet de juger certains délits, comme la piraterie aérienne, sur le territoire national où s'est effectué l'arrestation du ou des présumés responsables du délit, quel qu'en soit le lieu et quelle que soit la nationalité des accusés. La cour de Francfort, sous la présidence du juge Mückenberger, justifie sa compétence à juger le jeune Libanais, au regard des six chefs d'inculpation qui lui sont adressés ${ }^{8}$, sur la base d'une jurisprudence fondée sur des accords internationaux auxquels est liée la RFA. Les attendus de la cour de Hesse, qui condamne à la réclusion à perpétuité Mohamed Hamade, sont consacrés dans une très large mesure à l'âge de l'accusé. Ce point est capital dans la conduite du procès car il détermine la nature de la juridiction et donc la gravité de la peine encourue. Contrairement aux décisions juridiques prises en avril 1988 qui permettaient de juger le terroriste devant une cour pour mineurs, et qui confortaient ainsi la volonté de Bonn d'atténuer le mécontentement comme le durcissement du Hezbollah, le tribunal de grande instance de Francfort confirme sa compétence à juger le cas Mohamed Hamade. En effet lorsqu'un prévenu est âgé de 18 à 21 ans, les cours allemandes peuvent déterminer en fonction des aptitudes physiques, psychiques et intellectuelles de celui-ci sa capacité à assumer juridiquement un jugement de droit commun ou pénal. Après avoir comparé et recoupé une série de documents, la troisième grande chambre correctionnelle de Hesse arrête la date de naissance, jusqu'ici incertaine autant par stratégie de l'accusé que par imprécisions socioculturelles dues à l'identité musulmane chiite de ce dernier', au 13 juin 1964, date enregistrée auprès des autorités communales de Marjeyoum, au Sud Liban. Cependant, le tribunal émet la possibilité d'une erreur quant au jour de naissance mais ni au mois et à l'année. La marge d'incertitude a tout son poids car le détournement du Boeing américain s'est déroulé le 14 juin 1985. Mohamed Hamade venait-il d'avoir 21 ans ? dans cette hypothèse il ne peut plus relever de tribunaux pour mineurs, ou allait-il avoir 21 ans? A l'étude et à l'audition de nombreux témoins comme de l'accusé luimême, les juges de Francfort refuseront toute circonstance atténuante ou "exceptionnelle" dont aurait pu bénéficier le jeune Libanais. Sa maturité et sa responsabilité étant reconnues pleines et entières, l'imprécision du jour de sa naissance ne joue pas en faveur d'un changement de juridiction ou d'une réduction de peine.

Le déroulement du procès présente un triple intérêt stratégique. 
Au-delà des preuves avancées sur les agissements de Mohamed Ali Hamade au sein des cellules terroristes du Hezbollah, il met à plat le rôle-clé de ce mouvement dans l'organisation d'attentats. De multiples auditions et confrontations démontrent que ceux-ci sont fomentés en étroite collaboration avec le régime iranien, qui sert de soutien matériel, politique et idéologique, et la Syrie, qui apparait comme un passage obligé des terroristes. Le procès prouve aussi, et surtout pour la justice française, l'existence d'un réseau spécifique libanais d'inspiration iranienne, dirigé par Ali Fouad Saleh pour agir sur le territoire français.

Au cours de la procédure consacrée à la "vie et aux origines de l'accusé", Mohamed Hamade déclare être un "combattant du Hezbollah". Il suivra une formation militaire au sein de l'OLP, sera proche du groupe Amal, puis adhérera au Hezbollah. Il effectuera plusieurs séjours en RFA, en 1982, 83 et 84, où il sera hébergé par son frère Abbas et demandera le droit d'asile aux autorités allemandes qui le lui refuseront. En 1984, il retourne au Liban pour servir le mouvement chiite libanais, dont un des chefs de sécurité est Abdel Hadi Hamade. Le mot d'ordre du Hezbollah est de lutter contre l'" impérialisme", en particulier contre toutes les installations, ou représentations, françaises et américaines. En 1985, Mohamed travaille pour son frère, Abdel Hadi II reçoit alors une mission rémunérée, vraisemblablement celle du détournement du vol 847 de la TWA pour que Washington fasse pression sur les Israéliens afin de libérer plusieurs centaines de prisonniers arabes, détenus à la prison de Atlit. Après le détournement et le meurtre d'un ex-militaire américain, Mohamed se rend en juillet 1985 en Iran, via la Syrie. A son retour au Liban en 1986, il travaille à nouveau pour le Hezbollah mais sous les ordres d'un dénommé Tashin. Celui-ci commanditera à Mohamed et à Abbas le transport de matières explosives, destinées à la France, au réseau Ali Fouad Saleh. L'arrestation de Mohamed Hamade", à Francfort, le 13 janvier 1987, en possession d'un faux passeport au nom de "Youssef Rida" mais surtout avec l'équivalent de $11 \mathrm{~kg}$ de TNT, ouvre la voie à une longue enquête qui sera décisive pour la procédure française à l'encontre des activités terroristes du Libanais Saleh ${ }^{10}$.

Conclusion

Le traitement politique de l'affaire des otages allemands au Liban rend compte des mécanismes singuliers qui sous-tendent le développement de la diplomatie de la République fédérale d'Allemagne. A partir des contraintes historiques dont elle hérite en 1949, et qui lui interdisent toute ambition de puissance politique de premier plan, la RFA trouve rapidement une audience internationale dans le domaine économique. En s'appuyant sur une large plate-forme commerciale - la RFA s'est imposée en l'espace de quarante années comme la première puissance commerçante du monde, Bonn parvient à mettre en oeuvre une diplomatie de conciliation et à gérer des politiques parallèles envers des partenaires qui peuvent être des "frères ennemis", comme sur l'échiquier moyen-oriental.

Afin de répondre à ses intérêts multiples de nation marchande dominante, qui lui procurent et lui commandent un réseau relationnel particulièrement dense, et afin de respecter ses obligations historiques à l'égard d'Israël, la RFA cultive une politique de neutralité, voire de médiation, dans la région ${ }^{11}$. La diplomatie de Bonn repose sur trois atouts. Tout d'abord, les liens multilatéraux que lui assurent ses échanges commerciaux. Puis, l'absence de passé colonial et de leadership allemand au MoyenOrient qui préservent la RFA d'attaques à caractère nationaliste ou idéologique menées par des groupes comme le Hezbollah ${ }^{12}$. Enfin l'attirance d'une partie des élites 
iraniennes ou de certains pays arabes (Syrie, égypte, Irak) pour l'"Allemagne"13, qui permet à la République fédérale de s'assurer des amitiés politiques ou de (re)nouer avec des cercles dirigeants de pays d'Orient. Forte de connexions étroites, Bonn peut proposer ses "bons offices" et participer au règlement d'affaires comme celle des otages américains, détenus à l'ambassade des Etats-Unis à Téhéran en 1979, ou celle des otages occidentaux détenus au Liban depuis 1985. Le responsable de la diplomatie allemande intervient en effet pour les états-Unis et la Grande-Bretagne auprès des autorités de la République islamique. Hans-Dietrich Genscher s'appuie sur des réseaux officiels (diplomates, chargés de missions expérimentés), des réseaux traditionnels (les chambres de commerce ou les associations germano-arabes) et des réseaux privés (liens - souvent tissés sur la base d'accords commerciaux et donc d'intérêts mutuels - entre des hommes d'affaires ou politiques allemands et des responsables gouvernementaux au Moyen-Orient) pour prétendre à un exercice de négociation ou de médiation'9. Ainsi Sadegh Tabatabai, un homme clé du gouvernement de Khomeyni qui entretient des contacts réguliers avec des personnalités allemandes, sera un maillon essentiel des négociations triangulaires entre la RFA, l'Iran et les USA, en 1980, puis entre la Syrie, le Liban, l'Iran et la RFA pour les derniers otages occidentaux détenus à Beyrouth ${ }^{14}$.

Le traitement judiciaire de l'affaire Hamade reflète, quant à lui, la détermination des magistrats allemands à mener sans contraintes externes les procès des deux terroristes. Toutefois, le sort des frères libanais est lié, selon toute vraisemblance, à un compromis entre les ravisseurs, l'Iran et la Syrie, d'un côté et la RFA de l'autre. Pour des raisons politiques, le président fédéral Richard von Weizsäcker pourrait gracier à moyen terme Mohamed Hamade comme le fut Anice Naccache par François Mitterrand en juillet $1990^{15}$. L'enlèvement par le "clan" Hamade de quatre autres otages allemands en $1989^{16}$, opéré quelques jours avant l'issue du procès de Mohamed à Francfort dans le but de maintenir la pression sur les autorités politiques de Bonn, témoigne de la spécificité des prises d'otages qui touchent la République fédérale. Elles ne sont pas dirigées, comme celles qui ont frappé la France, la Grande-Bretagne ou les Etats-Unis, contre la puissance "impérialiste" ou "sioniste" que dénonce le Hezbollah, mais pour obtenir la libération des deux terroristes libanais arrêtés et condamnés en RFA Aussi la remise en liberté des derniers citoyens allemands ne dépend-elle pas directement des changements des rapports de force intervenus sur la scène du Moyen-Orient en 1991". Les frères Hamade pourrait néanmoins devenir un enjeu politique non négligeable pour la diplomatie de "paix" que se fixe la RFA. En effet "si la libération des deux Libanais pouvait être une contribution significative à une pacification d'un des conflits les plus inextricables du monde, alors la prise en considération de la protection des otages allemands doit peser dans la balance"17.

Annexe I Organigramme du réseau Ali Fouad Saleh et du clan Hamade

Ali Fouad Saleh ${ }^{18}$ Famille Hamade ${ }^{19}$

- Abdel Hadi ${ }^{20}$ : responsable du "parti de Dieu" et de l'enlèvement de Rudolf Cordes et de Alfred Schmidt.

Lié à Tashin, dont il serait le supérieur hiérarchique, ainsi que celui de Hassan Gohsn. Il leur aurait donné l'ordre de commettre les attentats de Paris.

- Mohamed: porteur d'explosifs liquides sur ordre d'un responsable du Hezbollah 
surnommé Tashin, membre du commando qui détourne le Boeing de la TWA en juin à Beyrouth et auteur de l'assassinat du passager américain Robert Stethem.

- Abbas : porteur d'explosifs liquides sur ordre de Tashin et acteur de l'enlèvement des deux ressortissants allemands.

- Famille Gohsn: organise une "cellule de crise" pour empêcher l'extradition de Mohamed Hamade vers les USA après son arrestation sur le territoire allemand en $1987^{21}$.

- Abdel Mehdi (père : trafiquant d'armes et de stupéfiants. En contact avec d'autres trafiquants, dont l'un des fils travaillant à l'ambassade de France à Beyrouth sera chargé d'obtenir des visas pour les membres du réseau Ali Fouad Saleh et de la famille Hamade. En outre, le numéro de téléphone de Abdel Mehdi sera retrouvé dans les carnets de Saleh et de Mohamed Hamade.

- Hassan (fils) : liens avec Ali Fouad Saleh et Mohamed Hamade. Participe aux attentats commis en 1985 et en 1986 à Paris. Joue un rôle primordial dans leur élaboration, avec la complicité de Ibrahim Ahil. Par ailleurs, Hassan a pris part à la réunion au cours de laquelle sera décidé l'enlèvement de R. Cordes et de A. Schmidt ainsi qu'à la préparation logistique de l'enlèvement de ce dernier par les frères Hamade.

- Ibrahim (fils : libéré d'une prison israélienne par l'entremise de Mohamed Hamade.

- Ali (fils) : du fait de son handicap physique et mental, et surtout du fait des liens existant entre la famille Gohsn et le Hezbollah (dont Saleh et les frères Hamade), son identité servira de prête-nom à Ibrahim Ahil.

- Hussein (fils) : marié à une s, ur Mahroun, dont l'un des oncles Rachid, jouera un rôle d'intermédiaire dans le processus de libération des otages allemands.

- Ibrahim Akil: alias "Ali" Gohsn et Tashin. Joue un rôle très important dans les activités du Hezbollah, tant en RFA qu'en France. Confie à Mohamed et Abbas Hamade la mission d'importer l'explosif liquide sur le territoire allemand avant sa destination finale: Paris (9 litres de nitrate de méthyle pour le premier, 12 pour le second). Participe aux attentats dans la capitale française avec Hassan Gohsn dans le cadre du réseau Saleh. Aurait également joué un rôle décisif dans la scission du mouvement Amal et aurait été le responsable des services secrets du Hezbollah commanditant des prises d'otages et des actes terroristes.

- Mohamed Mehdi (arrêté à Paris le 31.12.86) permet l'adhésion de Ali Fouad Saleh au Hezbollah. Lié à Abdel Hadi Hamade. Témoins principaux aux procès Ali Fouad Saleh à Paris, de Mohamed et de Abbas Hamade, respectivement à Francfort et Düsseldorf : Baha Mahroum, Kamal Habhab, Rachid Mahroun, Sami Slim²2.

Annexe II Otages occidentaux détenus au Liban entre 1985 et 1991 par le Hezbollah 1985

Cultures \& Conflits, 05 | printemps 1992 
Terry Anderson (USA) : directeur général de l'agence de presse américaine Associated Press, enlevé le 16 mars 1985 et libéré le 4 décembre 1991.

- Marcel Fontaine et Marcel Carton (France) : diplomates, enlevés le 22 mars, libérés le 4 mai 1988.

- Gilles Sydney Peyrolles (France) : directeur du centre culturel français de Tripoli (Liban nord), enlevé le 23 mars 1985 et libéré le 2 avril 1985.

- Michel Seurat et Jean-Paul Kauffmann (France) : respectivement, chercheur au CNRS et journaliste à l'événement du Jeudi enlevés le 22 mai 1985. Michel Seurat décède au cours de sa détention, Jean-Paul Kauffmann est libéré le 4 mai 1988.

Thomas Sutherland (USA) : doyen de la faculté d'agronomie de

l'AUB (université américaine de Beyrouth), enlevé le 9 juin 1985 et libéré le 18 novembre 1991.

- Alberto Molinari (Italie) cadre commercial, enlevé le 11 septembre 1985 et disparu. 1986

- Brian Keenan (lrlande) : enseignant, enlevé le Il avril 1986 et libéré au printemps 1990.

- John Mac Carthy (GB) : cameraman, enlevé le 17 avril 1986 et libéré le 8 août 1991.

Marcel Coudari (France) : enlevé le 24 février 1986 et libéré le 11 novembre 1986.

- Philippe Rochot, Georges Hansen, Aurel Cornéa et Jean-Louis Normandin (France) : journalistes à Antenne 2, enlevés le 8 mars 1986 et libérés respectivement, pour les deux premiers otages, le 20juin 1986, puis le 24 décembre 1986 et le 27 novembre 1987. Camille Sontag (France) : enlevé le 7 mai et libéré le 11 novembre 1986.

- Frank-Herbert Reed (USA) : directeur de la Lebanese International School (LIS), enlevé le 9 septembre 1986 et libéré le 20 avril 1990.

- Joseph Cicippio (USA) : comptable de l'université américaine de Beyrouth (AUB), enlevé le 12 septembre 1986 et libéré le 2 décembre 1991.

- Edward Tracy (USA) : écrivain, enlevé le 21 octobre 1986 et libéré le11 août 1991. 1987

- Roger Auque (France) : journaliste, enlevé le 13 janvier 1987 et libéré le 27 novembre 1987.

- Rudolf Cordes (RFA) : cadre commercial du groupe chimique

Hoechst, enlevé le 17 janvier 1987 et libéré le 12 septembre 1988. Alfred Schmidt (RFA) : technicien de la firme Siemens, enlevé le 20 janvier 1987 et libéré le 7 septembre 1987. 
- Terry Waite (GB): envoyé spécial de l'archevêque de Canturbury et principal médiateur dans l'affaire des otages occidentaux, enlevé le 20janvier 1987 et libéré le 18 novembre 1991.

Jesse Turner, Alan Steen et Robert Polhill (USA) : enseignants du Beirut Universíty College (BUC), enlevés le 24 janvier 1987 et libérés, respectivement, le octobre 1991, le 3 décembre 1991 et au printemps 1990.

1988

- William Higgins (USA) : commandant en chef adjoint de

l'ONUST (Organisation des Nations-Unies pour la supervision de la trêve), enlevé le 17 février et disparu.

1989

- Jack Mann (GB) : ancien pilote de la Royal Air Force, enlevé le 12 mai 1989 et libéré le 24 septembre 1991.

- Petra Schnitzler, Heinrich Strübig et Markus Quint (RFA) : membres de l'association humanitaire

"Asme Humanitas", enlevés le 4 mai 1989 et libérés, respectivement le 5 mai 1989 et le 14 mai 1989, pour le dernier.

- Heinrich Strübig et Thomas Kemptner (RFA) : membres de

"Asme Humanitas", enlevés le 16 mai 1989.

Otages occidentaux disparus

- Nicolas Kluiters : prêtre néerlandais retrouvé assassiné le 1er avril 1985.

- Dennis Hill universitaire britannique, assassiné le 29 mai 1985.

- Arkadi Katkov diplomate soviétique, assassiné le 2 octobre 1985.

William Buckley : troisième secrétaire de l'ambassade des Etats-Unis à Beyrouth, mort annoncée par ses ravisseurs le 4 octobre 1985.

- Leigh Douglas et Philip Patfeld : enseignants britanniques, retrouvés assassinés le 17 avril 1986.

- Peter Kilburn : universitaire américain, retrouvé assassiné le 17 avril 1986.

Alec Collett : journaliste britannique et fonctionnaire de L'UNRWA, mort annoncée par ses ravisseurs le 17 avril 1986.

- Michel Seurat : ehercheur français du CNRS, mort annoncée par ses ravisseurs, qui l'auraient tué, début mars 1986. Marie Seurat écrit, dans son ouvrage les Corbeaux d'Alep, qu'il serait décédé au cours de sa détention, fin 1985. 
- Alberto Moliani : cadre commercial italien.

- Lt-Colonel William Higgins.

\section{NOTES}

1. Outre les missions de "réconciliation" entre les pays arabes et la RFA en période de crises en 1965, ou de tensions au début des années 70, H.J. Wischnewski est chargé de résoudre les prises d'otages lors du détournement d'un avion de la Luftlhansa à Modagiscio et à l'aéroport de Bonn-Cologne.

2. Le chef religieux du mouvement Hezbollah, le Cheikh Mohammed Hussein Fadlallah confie à un interlocuteur allemand que "la clé de cette affaire réside exclusivement à Téhéran", cf. Frankfurter Runschau, 24.06.89.

3. Voir note 40.

4. Cette aide au développement est structurée en trois paries. Une enveloppe de 55,9 millions de DM est destinée à l'acquisition de biens divers. Une seconde de 14,7 millions au financement d'un complexe d'extraction pétrolière. Enfin, la troisième de 75,5 est liée à l'évolution du problème des otages allemands. Cf. Hamburger Morgenpost et AP septembre 1987.

5. Cependant, selon les sources de la presse occidentale ou moyen-orientale, le montant des rançons varie de un à trois millions de dollars.

6. Le Monde, Reuter, 28.01 .88 .

7. L'arrestation, en RFA, de cette citoyenne allemande est opérée dans le cadre de l'enquête sur l'attentat à la Bombe d'avril 1986 contre ln discothèque de Berlin-ouest, "La belle".

8. Meurtre, détournent d'avion, prise d'otages, coups et blessures, port illégal de matières explosives et falsification de documents.

9. Un expert sur le monde arabe et musulman sera consulté et entendu par le tribunal afin de savoir si le fait de ne pouvoir déterminer avec exactitude, au jour près mais aussi au mois, voire dans certains cas, à l'année près, une date de naissance relève de moeurs ou de pratiques courantes. La réponse sera affirmative

10. Voir annexe I.

11. Lors de la guerre Iran-Irak, la RFA chercha continuellement, dans les limites que la solidarité avec ses alliés européens et américains impose, à conserver une attitude réservée. En effet, tenter de transformer son influence commerciale en influence politique, dans une région instable et minée par de multiples conflits, serait risquer de compromettre à la fois ses échanges commerciaux et la confiance des pays du Moyenorient.

12. Frankfurter Allgemeine, "In Beirut haben die Deutschen bis zuletzt ausgeharrt Schutz durch die Bonner Nahost Politik", 29.01.87.

13. Se concrétisant souvent par un cursus universitaire entrepris en RFA. 
14. . Sadegh Tabatabai est le beau-frère d'un des fils de Khomeyni. Il a entrepris une partie de ses études en RFA où il a présidé une association représentant les étrangers à l'université. Ces responsabilités lui ont permis de nouer des liens avec de futures personnalités politiques allemandes et appartenant nu SPD (Dieter Schinzel) et des juristes comme Peter Heinemann, fils de l'ancien chancelier Gustav Heinemann, qui fut 1 'auocat-conseil du gouvernement iranien pour le litige concernant le blocage des comptes de la République islamique par les banques américaines. Voir Chrislophe Warren, e.a, American Hostages in Iran. The Conduct of a crisis, New Haven, 1985, pp. 235-280 et 297-307.

15. Cette grâce présidentielle allemande pourrait également répondre à une politique d'ouverture du gouvernement fédéral à l'égard des terroristes de la RAF arrêtés et condamnés pour la plupart à la réclusion à perpétuité (cf. Frankfurtcr Allegemeine, 09.01.92). Richard von Weizsäcker a déjà usé de son droit de grâce pour la terroriste Angelika Speitel. Jugée le 30 novembre 1979 à la réclusion à vie, elle retrouve la liberté le 30juin 1990 pour "s'être repentie avec sincérité de ses actes".

16. Petra Schnitzler Heinrich Strübig et Marhus Quint sont enlevés le 4 mai 1989 au Liban. Les deux premiers sont libérés le 5 mai, le dernier le 14. Heinrich Strübig et Thomas kemptner sont enlevés le 1er mai 1989. Ils travaillent pour l'association humanitaire "Asme Humanitas".

17. Michael Bothe, "Bendenhliches und Nachdenkliches - soll einen Tausch der Hamadi Brüder gegen deutsche Geiseln im Libanon geben ?" in Tageszeitung, 05.12.91.

18. Voir Didier Bigo, op., cit. Ali Fouad Saleh dirige à Paris un réseau libanais, d'inspiration iranienne. Il sera responsable des attentats commis en France en $19 \& 5$ et 1986 à l'initiative du Hezbollah. Né à Paris, le 10 mai 1958, mais de nationalité tunisienne, il passe toute son enfance à Tunis jusqu'à son départ pour l'Iran en 1982. Il effectue différents séjours dans la ville sainte de Qom où il y rencontrera Vahid Gordji, attaché à l'ambassade d'Iran à Paris, mais surtout chargé par Téhéran de négocier avec la France dans l'affaire des otages. Par la suite, Saleh se liera au Hezbollah par le biais de rencontres multiples avec des membres de cette organisation : Mohamed Mehdi, Alaa Alaeddine, Sami Slim.

19. Le "clan" Hamade est lié de près au Jihad islamique pour la libération de la Palestine, à la Cellule révolutionnaire arabe-Brigade Omar Muchtar, ou encore à l'Organisation révolutionnaire des musulmans socialistes.

20. . Abdel Hodi Hamade est considéré par les services de renseignements occidentaux comme l'un des trois chefs de guerre du Hezbollah avec Hadj Imad Fayez Moughnieh et Mohamed Aidar. Il exerce des fonctions importantes dans le domaine de la sécurité du mouvement et est le responsable du secteur de Bourj el Brajneh. Abdel Hadi Hamade avait d'abord été proche du mouvement chiite Amal, dirigé par Nabih Berri, avant d'adhérer au Hezbollah, mouvement islamique fondamentaliste sous influence iranienne, issu d'une scission d'Amal. Par ailleurs, il entretient des relations non seulement étmites mais aussi hiérarchiques avec plusieurs membre,s du réseau de Ali Fouad Saleh.

21. Outre la famille Gohsn, qui est à l'origine de cette réunion, Abdel Hadi Hamade et Ibrahim Akil, alias Tashin et "Ali" Gohsn, prennent part aux décisions qui y sont prises. 22. Bahn Mahroun est né à Beyrouth en 19fi8 et a effectué plusieurs séjours en RFA de 1983 à 1987. Il déclare bien connaître la famille Gohsn avec laquelle il a des liens de parenté. Une de ses s urs a Epousé Hussein Gohsn. De plus, Baha Mahroun est le neueu rle Rachid Mahroun qui $n$ tenu le rôle d'in1erntEdinire entre la famille Hamade et le 
gouvernement de Bonn pour la libération de Mohamed et Abbas en échange des otages allemands. Kamal Habhab, mémoire de l'Organisation communiste des travailleurs a élé enlevé en février 1986 par le Hezbollah. Au cours des procès Hamade, il témoigne sur Tashin, responsable de son enlèvement.

\section{RÉSUMÉS}

L'enlèvement de deux citoyens allemands au Liban en 1987 puis de quatre autres en 1989, relève d'une problématique singulière, différente de celle concernant les pays occidentaux victimes également de prises d'otages. Le mouvement du Hezbollah demeure certes l'auteur principal de toutes ces actions terroristes mais, dans le cas de la RFA, il s'agit moins pour lui de porter atteinte à la présence allemande au Moyen-Orient que de chercher à exercer un chantage humain sur les autorités politiques de Bonn afin qu'elles libèrent Mohamed et Abbas Hamade, arrêtés à Francfort en possession d'explosifs. Aussi la singularité des motifs qui commandent l'enlèvement de citoyens de la République fédérale met-elle en relief la spécificité de la diplomatie allemande dans la région. En reposant sur des intérêts économiques et des particularismes historiques bien définis, elle bénéfice d'un certain nombre d'atouts politiques pour prétendre à des négociations rapides.

The kidnapping of two German citizens in Lebanon in 1987, followed by that of four more in 1989, have their own specificity which differs from the taking of many other Western hostages. While Hezbollah undoubtedly is the main force behind these terrorist acts, in the case of the Federal Republic, it is not so much a question of inflicting damage on German interests in the Middle East, as of blackmailing Bonn authorities into releasing Mohamed and Abbas Hamade, arrested in Frankfurt in possession of explosives. Thus the motivations behind the kidnapping of citizens of the German Federal Republic reflect the role of German diplomacy in the region. Economic interests and specific historical factors provide the setting supposed to facilitate rapid negotiations.

\section{INDEX}

Index géographique : Liban

Mots-clés : coopération internationale, gestion de crise

Thèmes : Hezbollah

\section{AUTEUR}

\section{SYLVIE LEMASSON}

Chercheur associé au CERI 